\title{
Resultados de una obra construida de hormigón con aire incorporado
}

Carlos Arcos d'Haịnaut, investigador - IDIEM

$684-8$

sinopsis

Este trabajo presenta el análisis de los resultados de una obra construida en su totalidad de hormigón con aire incorporado. Los datos analizados corresponden a los resultados obtenidos durante el control de la obra, y se encuentran en certificados oficiales emitidos por IDIEM. Se han logrado algunas relaciones generales entre los factores y efectos de los componentes en un hormigón con aire incorporado que refiejan las condiciones de trabajo usuales, y se pueden extender a los hormigones con aire incorporado en general.

\section{LA OBRA}

La obra es un frigorífico construido en Valparaíso, Chile. Sus instalaciones comprende un edificio de hormigón armado con superficie de $7.700 \mathrm{~m}^{2}$ distribuidos en dos plantas, y un volumen total de hormigón de $10.000 \mathrm{~m}^{3}$. Consta de seis cámaras frigorificas con capacidad total de almacenamiento de 380.000 cajas de manzanas o 4.100 toneladas de carne congelada 0 sus equivalentes. Las especificaciones exigian una resistencia mínima en probeta cúbica de $20 \mathrm{~cm}$ de arista a 28 dias de $300 \mathrm{kgf} / \mathrm{cm}^{2}$, y un porcentaje de aire incorporado de $4 \%$ para todos los hormigones, excepto para el hormigón de sobrelosa que estará expuesto sin protección ni aislación al ambiente de las cámaras frigorificas. Para éstos se fijaba un $6 \%$ de aire incorporado. Las temperaturas extremas de funcionamiento de las cámaras son de $+15^{\circ} \mathrm{C}$ y $-25^{\circ} \mathrm{C}$.

\section{CONTROL DEL HORMIGON CON AIRE INCORPORADO}

\subsection{MATERIALES}

La grava es un árido mixto de procedencia fluvial elaborado en planta de tamaño máximo 2". La arena también es de procedencia fluvial y de granulometria relativamente constante con poca variación del contenido de árido grueso. La medición de los áridos se hacía en volumen. Durante la obra se efectuó control de los áridos con análisis completo de 24 muestras de grava y 19 de arena, lo que da una muestra analizada por cada $300 \mathrm{~m}^{3}$ de material. Los resultados del control de los áridos se presentan en las Tablas I y II y Figuras 1 y 2.

El cemento usado en obra fue un "portland con agregado tipo A" que viene a ser equivalente a un portland puzolánico. Es de grado alta resistencia, salvo en el primer período que correspondió a un grado corriente. El cemento envasado en sacos de $42,5 \mathrm{~kg}$ se dosificaba en peso por sacos completos.

El agua empleada en los hormigones era potable.

El aditivo incorporador de aire fue Fro-Be de Sika que se emplea diluido en el agua de amasado en proporción recomendada por el fabricante entre 0,3 y $1,0 \%$ del peso del cemento.

\subsection{EQUIPO}

Las betoneras son de eje horizontal de capacidad $0,25 \mathrm{~m}^{3}$ y el aerímetro es Techkote Air Meter del tipo medidor por presión.

\section{HORMIGON}

El muestreo del hormigón se realizaba de acuerdo a la norma, y se determinaban sus caracteristicas como hormigón fresco, contenido de aire, y se confeccionaban probetas para ser ensayadas a compresión.

Los resultados del control del aire incorporado se presentan en la Tabla III y Figuras 3 y 4 . 
TABLA 1 : Grava - granulometrías - 24 muestras

\begin{tabular}{|c|c|c|}
\hline \multirow{2}{*}{$\therefore \quad$ Tamiz } & \multicolumn{2}{|c|}{ Granulometría, \% pasa } \\
\hline & Media & Desviación \\
\hline $2^{\prime \prime}$ & 100,0 & - \\
\hline . $\quad 11 / 2^{\prime \prime}$ & 93,6 & 4,4 \\
\hline $1^{\prime \prime}$ & 59,6 & 12,5 \\
\hline $3 / 4^{\prime \prime}$ & 28,0 & 7,8 \\
\hline $1 / 2^{\prime \prime}$ & 4,7 & 3,0 \\
\hline $3 / 811$ & 2,2 & 2,2 \\
\hline ASTM N $N 4$ & 1,3 & 1,9 \\
\hline $\begin{array}{l}\text { Densidad aparente, } \\
\mathrm{kgf} / \mathrm{dm} 3\end{array}$ & 1,67 & 0,04 \\
\hline $\begin{array}{l}\text { Peso específico, } \\
\mathrm{kgf} / \mathrm{dm}^{3}\end{array}$ & 2,63 & 0,04 \\
\hline Arcilla, \% & 0,41 & 0,34 \\
\hline
\end{tabular}

TABLA II : Arena - granulometrías - 19 muestras

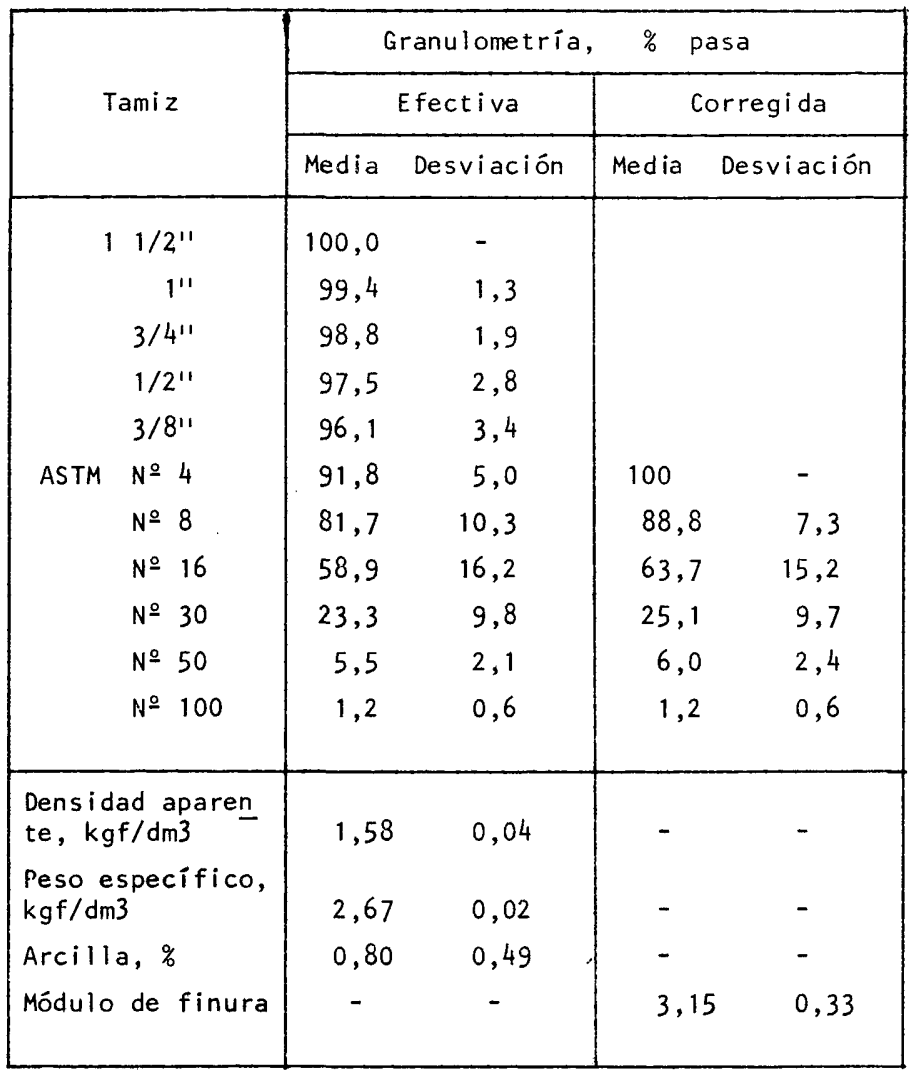

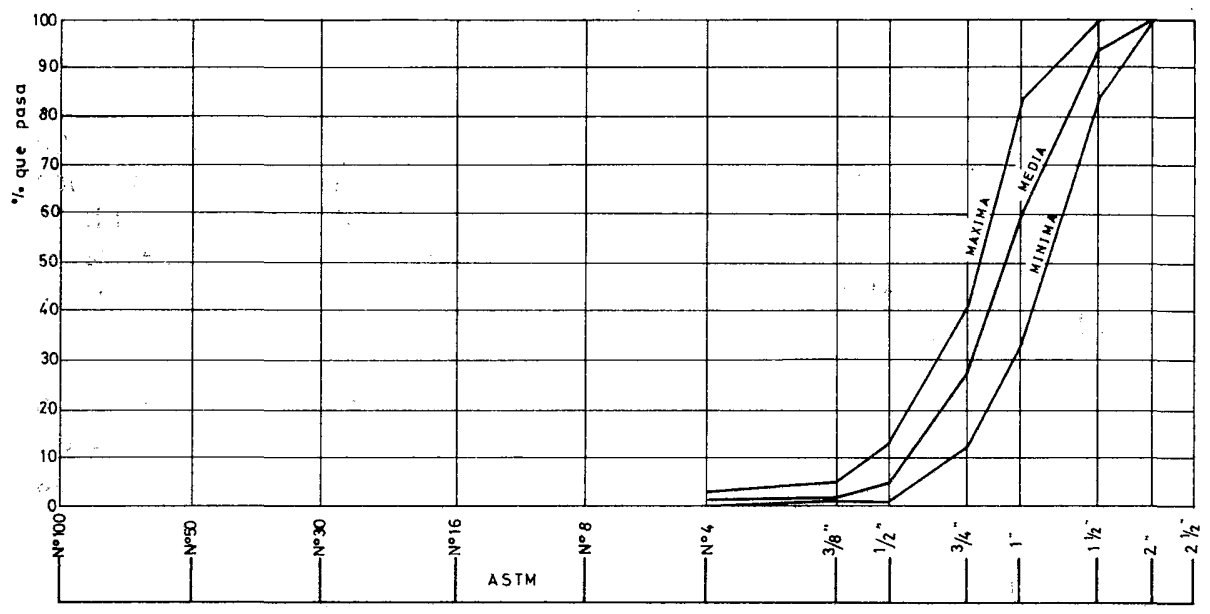

Fig. 1.-Grava -represen tación gráfica granulome trias- 24 muestras.

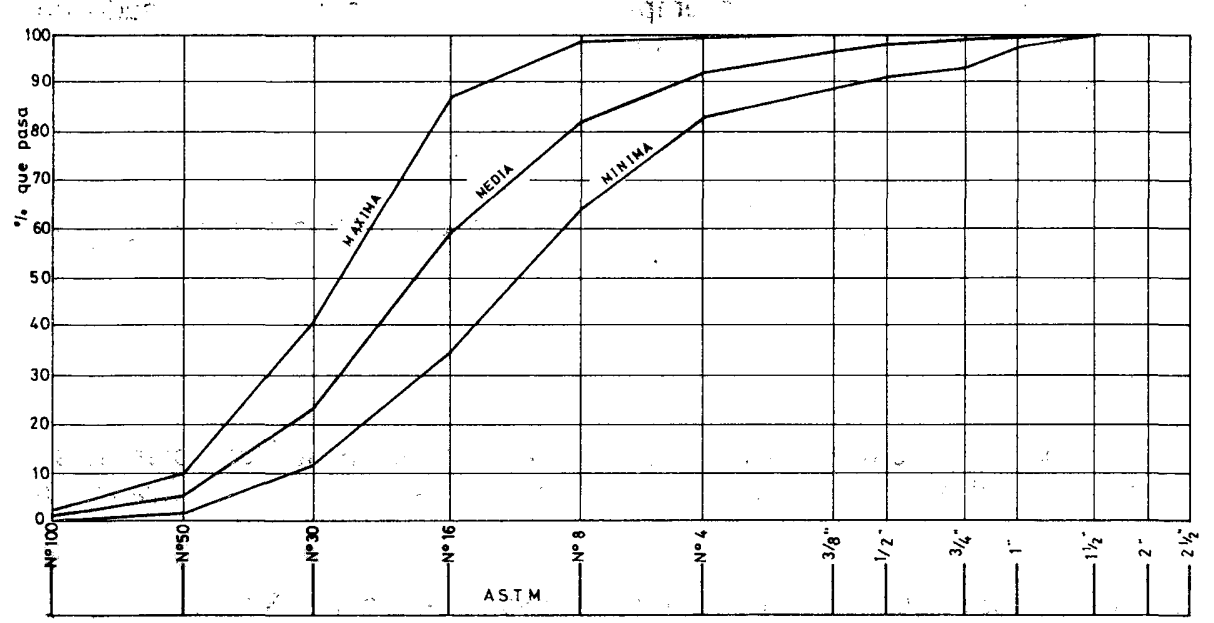

Fig. 2.-Arena - representación grafica granulometria efectiva - 19 muestras. 
La docilidad del hormigón se midió por el asentamiento en el cono de Abrams y sus resultados se presentan en las Tablas IV y $\mathbf{V}$ y Figura 5.

Cada muestra de hormigón constaba de tres probetas cúbicas que se ensayaban 1 a 14 dias y 2 a 28 dias. El curado era húmedo por medio de arpilleras mojadas. Al ensayo, las probetas se encontraban en estado seco al aire y se realizaba a una velocidad de $10 \mathrm{~mm} / \mathrm{min}$.

\section{RESULTADOS DE OBRA}

\subsection{RESULTADOS DEL ENSAYO A COMPRESION. CONTROL DE OBRA}

Durante la construcción de la obra se controló el hormigón por 334 muestras de hormigón distribuidas como sigue:

- 6 muestras de hormigón sin aire incorporado;

- 255 muestras de hormigón de $4 \%$ de aire incorporado especificado;

- 74 muestras de hormigón de $6 \%$ de aire incorporado especificado.

Esto significa haber controlado el hormigón con una muestra cada $30 \mathrm{~m}^{3}$ de hormigón aproximadamente. Los resultados de resistencia a compresión se han analizado considerando la especificación de aire incorporado y agrupados en períodos separados al cambiar el tipo y la dosis de cemento y de tener siempre un número de valores superior a 30 . En el caso de los hormigones de $6 \%$ de aire incorporado especificado por ser un número de valores más reducido, los períodos resultan inevitablemente de pocos valores, pero en ningún caso inferior a 14. La fracción defectuosa estimada es de $20 \%$. Los resultados del análisis estadistico se presentan en las Tablas VI y VII.

TABLA |II: Aire incorporado en el hormi gón

\begin{tabular}{|l|c|c|}
\hline \multirow{2}{*}{ Número de determinaciones } & Hormigón especificado con \\
\cline { 2 - 3 } & $4,0 \%$ de aire & $6,0 \%$ de aire \\
Aire incorporado medio, $\%$ & 253 & 74 \\
Desviación, \% & 3,86 & 6,02 \\
Coeficiente de variación, \% & 0,53 & 0,27 \\
\end{tabular}
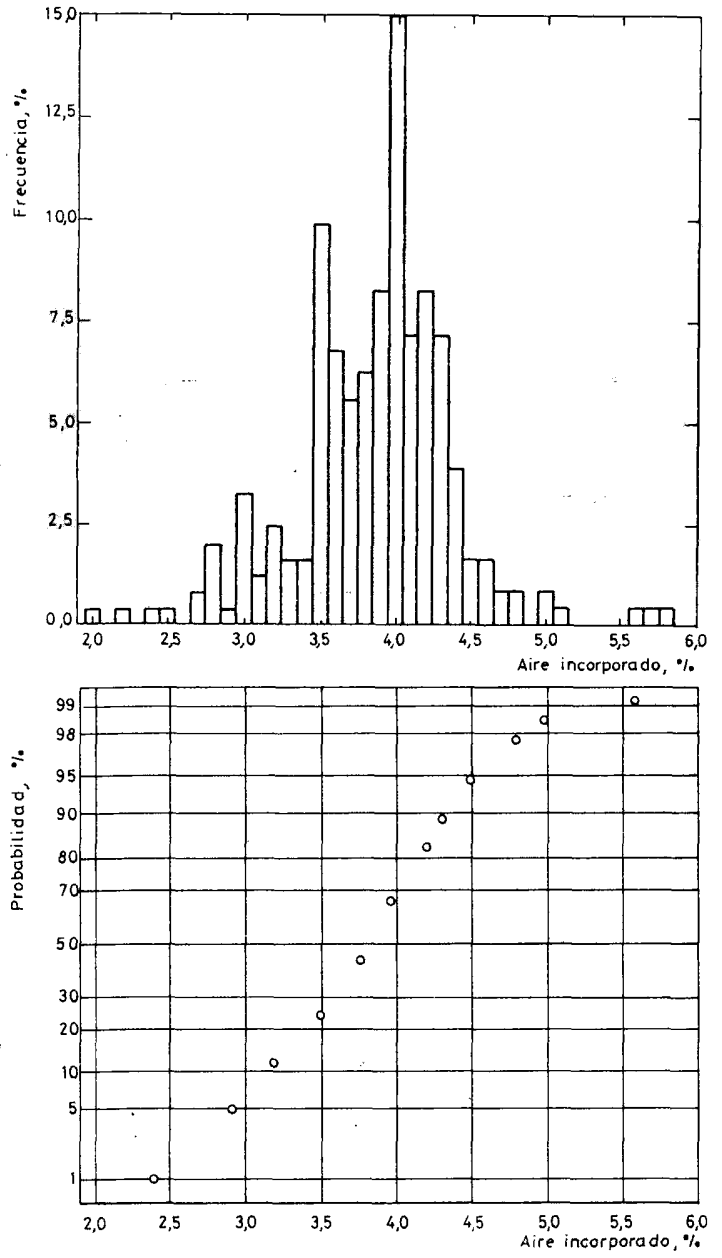

FIG. 3

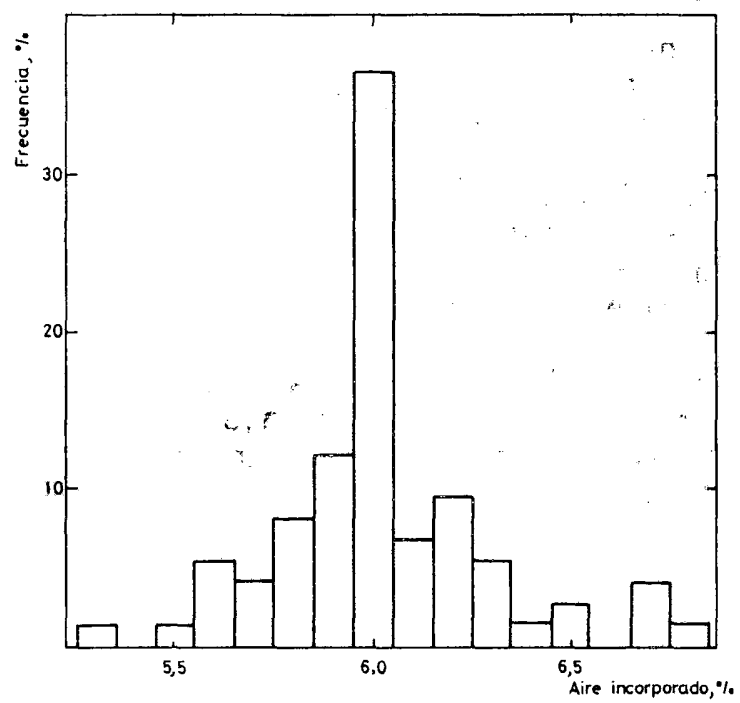

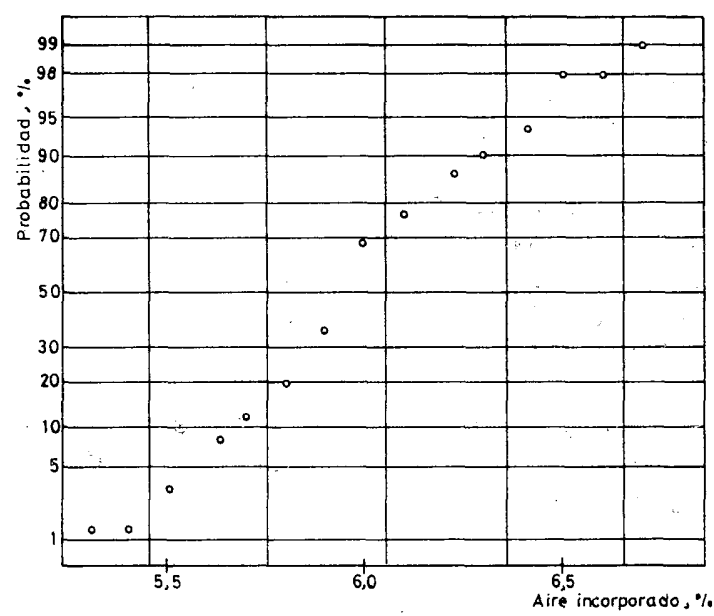

FIG. 4 
Entre otras consideraciones se puede desprender que en general con un buen control, dispersión baja o muy baja, es posible cumplir la especificación de $300 \mathrm{kgf} / \mathrm{cm}^{2}$ y $4 \%$ de aire incorporado con cemento grado alta resistencia y en dosis de $425 \mathrm{kgf} / \mathrm{m}^{3}$. En el caso de hormigones de $6 \%$ de aire incorporado especificado es posible cumplir la resistencia minima con dosis no inferiores a 488 $\mathrm{kgf} / \mathrm{m}^{3}$, cemento grado alta resistencia y dispersiones muy bajas. Por otra parte hay que hacer notar que para una obra con dosificación en volumen la dispersión alcanzada es baja llegando a valores de desviación estándar de $23 \mathrm{kgf} / \mathrm{cm}^{2}$ y coeficiente de variación de $7 \%$.

TABLA IV: Asentamiento Cono de Abrams

$\begin{array}{lr}\text { Número de determinaciones } & 334 \\ \text { Asentamiento medio, cm } & 6,51 \\ \text { Desviación, cm } & 1,47 \\ \text { Coef. de variación, \% } & 23,1\end{array}$

3.2. RESULTADOS DEL ENSAYO A COMPRESION. CONTROL DE LABORATORIO

TABLA V: Distribución de asentamientos Cono de Abrams

\begin{tabular}{|l|l|cc|}
\hline \multirow{2}{*}{$\begin{array}{c}\text { Banda de asenta } \\
\text { miento, cm. }\end{array}$} & \multicolumn{1}{|c|}{$\begin{array}{c}\text { Aspecto de } \\
\text { hormigón }\end{array}$} & Frecuencia & relativa \\
\cline { 3 - 4 } & & Número & $\%$ \\
\hline $\begin{array}{l}\text { Menor de } 4 \\
4 \text { a } 9\end{array}$ & seco-plástico & 3 & 1 \\
9 & plástico & 313 & 94 \\
blando & 18 & 5 \\
\hline
\end{tabular}

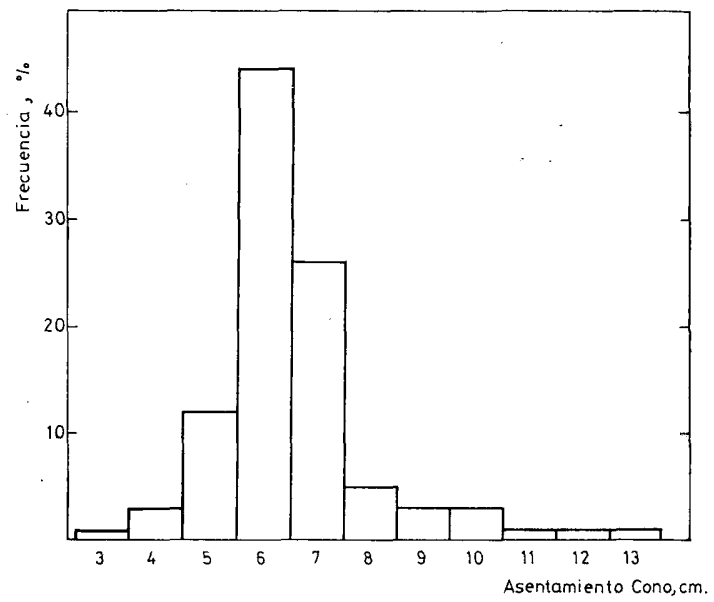

Los resultados del control de laboratorio son útiles para calificar el grado de control ejercido por éste en la obra. La uniformidad de los ensayos sobre probetas compañeras permiten calificar al laboratorio. Los promedios totales alcanzados en la obra, considerando una resistencia media de $320 \mathrm{kgf} / \mathrm{cm}^{2}$, son de $6,6 \mathrm{kgf} / \mathrm{cm}^{2}$ de rango medio, desviación estándar entre probetas compañeras de $5,85 \mathrm{kgf} / \mathrm{cm}^{2}$, lo que equivale a un ceoficiente de variación entre probetas compañeras de $1,82 \%$. El ACl-214-77 califica este control de laboratorio en obra como excelente.

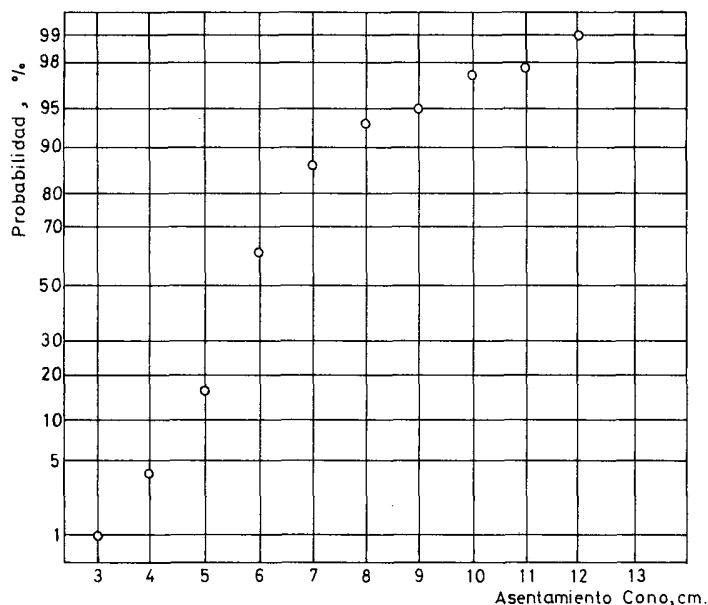

FIG. 5

\subsection{RELACION ENTRE ASENTAMIENTO EN EL CONO DE ABRAMS Y RESISTENCIA A COMPRESION DE HORMIGONES}

El asentamiento en el cono de Abrams es un ensayo fácil y posible de realizar en cualquier obra de hormigón. Nos señala ciertos aspectos de trabajabilidad y constitución reológica de la mezcla recién amasada. La trabajabilidad depende entre otros de la composición granulométrica, de la dosis de cemento, de las proporciones de la mezcla, de la forma y textura superficial de los granos de árido, del contenido de aire y por sobre todo de la cantidad de agua contenida en el hormigón.

En una obra, si se mantiene constante la dosis de cemento y dentro de ciertos limites se mantienen los demás factores mencionados, podemos suponer que el asentamiento está relacionado con la razón agua-cemento. 
TABLA VI: Resultados análisis hormigones de 4,0\% de aire incorporado especificado.

\begin{tabular}{|c|c|c|c|c|c|c|c|c|c|}
\hline Período & $\begin{array}{l}\text { Dos is, } \\
\text { grado }\end{array}$ & $\begin{array}{r}\mathrm{kgf} / \mathrm{m} 3 \\
\text { cemento }\end{array}$ & $N$ & $\underset{\mathrm{kg}}{\mathrm{Rm} / \mathrm{cm} 2}$ & $\begin{array}{c}\mathrm{S} \\
\mathrm{kgf} / \mathrm{cm}^{2}\end{array}$ & $\begin{array}{l}C \\
\%\end{array}$ & $\begin{array}{c}\mathrm{Rc} \\
\mathrm{kgf} / \mathrm{cm}^{2}\end{array}$ & $\begin{array}{r}\theta r \\
\%\end{array}$ & $\begin{array}{l}\mathrm{Am} \\
\mathrm{cm}\end{array}$ \\
\hline $\begin{array}{l}1^{\circ} \\
2^{\circ} \\
3^{\circ} \\
4^{\circ} \\
5^{\circ} \\
6^{\circ} \\
7^{\circ}\end{array}$ & $\begin{array}{l}380 \\
425 \\
425 \\
425 \\
425 \\
380 \\
425\end{array}$ & $\begin{array}{l}\text { cte } \\
A R \\
A R \\
A R \\
A R \\
A R \\
A R\end{array}$ & $\begin{array}{l}41 \\
36 \\
38 \\
34 \\
33 \\
42 \\
31\end{array}$ & $\begin{array}{l}281 \\
323 \\
331 \\
330 \\
360 \\
299 \\
315\end{array}$ & $\begin{array}{l}31,3 \\
35,8 \\
30,3 \\
23,0 \\
29,6 \\
31,7 \\
26,8\end{array}$ & $\begin{array}{r}11,2 \\
11,1 \\
9,1 \\
7,0 \\
8,2 \\
10,6 \\
8,5\end{array}$ & $\begin{array}{l}255 \\
293 \\
306 \\
311 \\
335 \\
272 \\
292\end{array}$ & $\begin{array}{r}>40 \\
26 \\
16 \\
10 \\
3 \\
>40 \\
29\end{array}$ & $\begin{array}{l}6,3 \\
6,9 \\
7,5 \\
6,5 \\
5,8 \\
6,1 \\
6,3\end{array}$ \\
\hline
\end{tabular}

TABLA VII: Resultados análisis hormigones de 6,0\% aire incorporado especificado.

\begin{tabular}{|c|c|c|c|c|c|c|c|c|c|}
\hline Período & $\begin{array}{l}\text { Dos is, } \\
\text { grado }\end{array}$ & $\begin{array}{c}\mathrm{kg} / \mathrm{m} 3 \\
\text { cemento }\end{array}$ & $N$ & $\mathrm{Rm}_{\mathrm{kgf} / \mathrm{cm}^{2}}$ & $\underset{\mathrm{kgf} / \mathrm{cm}^{2}}{ }$ & $\begin{array}{l}C \\
\%\end{array}$ & $\begin{array}{c}\mathrm{Rc} \\
\mathrm{kgf} / \mathrm{cm}^{2}\end{array}$ & $\begin{array}{l}3 r \\
\%\end{array}$ & $\begin{array}{l}\mathrm{Am} \\
\mathrm{cm}\end{array}$ \\
\hline $\begin{array}{l}1^{\circ} \\
2^{\circ} \\
3^{\circ} \\
4^{\circ}\end{array}$ & $\begin{array}{l}488 \\
446 \\
468 \\
488\end{array}$ & $\begin{array}{l}A R \\
A R \\
A R \\
A R\end{array}$ & $\begin{array}{l}14 \\
20 \\
16 \\
24\end{array}$ & $\begin{array}{l}346 \\
298 \\
296 \\
302\end{array}$ & $\begin{array}{l}38,8 \\
33,4 \\
30,2 \\
28,2\end{array}$ & $\begin{array}{r}11,2 \\
11,2 \\
10,2 \\
9,3\end{array}$ & $\begin{array}{l}312 \\
269 \\
270 \\
278\end{array}$ & $\begin{aligned} & 12 \\
& \gg 40 \\
& \gg \\
&>40\end{aligned}$ & $\begin{array}{l}6,3 \\
6,6 \\
6,6 \\
6,5\end{array}$ \\
\hline
\end{tabular}

NOTAS: $\quad \begin{aligned} & \mathrm{N}: \text { número de valores } \\ & \mathrm{Rm}: \text { resistencia media } \\ & \mathrm{S}: \text { desviación típica } \\ & \mathrm{C}: \text { Coef. de variación. }\end{aligned}$

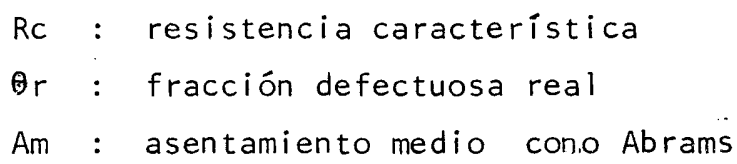

Ahora, si consideramos la fórmula de Abrams que determina la resistencia a compresión:

$$
R=\frac{M}{B^{w / c}}
$$

en que $\quad R$ : resistencia a compresión del hormigón

$\mathrm{W} / \mathrm{c}$ : razón agua-cemento en volúmenes

M y B: coeficientes que dependen de la calidad del cemento y edad del hormigón.

Nuestra relación se puede anotar como:

$$
\mathrm{R}_{28}=\frac{\mathrm{P}}{\mathrm{Q}^{A s}}
$$

en que $R_{28}$ : .: resistencia a compresión a 28 dias, $\mathrm{kgf} / \mathrm{cm}^{2}$

As: asentamiento en el cono de Abrams, cm

$P$ y Q: constantes.

A los datos hemos ajustado una recta de regresión por el método de minimos cuadrados. Se han considerado 175 valores que corresponden a hormigones fabricados con dosis de cemento de $425 \mathrm{kgf} / \mathrm{m}^{3}$. De ellos resulta la siguiente relación: 


$$
R_{28}=\frac{378,14}{1,0203^{A s}}
$$

y la expresión se ha dibujado en la Figura 6 en la que se han representado también todos los puntos.

Se puede observar claramente la gran dispersión en cada intervalo de la variable independiente, por lo que se ha calculado sólo la curva que indica el valor medio más probable, no así los límites de confianza, los que se encontrarian bastante alejados de la curva. Hay que considerar que el $70 \%$ de los valores pertenecen a asentamientos de 6 y $7 \mathrm{~cm}$.

Sin embargo, esta curva nos indica que existe una disminución de la resistencia a compresión del hormigón, aproximadamente de $6 \circ 7 \mathrm{kgf} / \mathrm{cm}^{2}$ por cada centímetro de aumento en el asentamiento. Queremos mostrar también que en una obra de esta naturaleza, en que todos los hormigones se fabricaron con caracteristicas similares a cemento y áridos, existe para cada valor del asentamiento, un valor de resistencia problable, más menos un error de importante magnitud. Esto significa en otras palabras, que de una muestra de hormigón tomada individualmente no se debe considerar el asentamiento en el cono de Abrams para estimar la resistencia a compresión del hormigón.

\subsection{RELACION ENTRE DOSIS DE ADITIVO Y CANTIDAD DE AIRE INCORPORADO EN EL HORMIGON}

La cantidad de aire incorporado varía entre otros factores con la dosis de cemento, la razón agua-cemento, los áridos y la dosis de aditivo. Para obtener la relación entre la cantidad de aire incorporado al hormigón y la dosis de aditivo se han considerado los resultados correspondientes a hormigones confeccionados con dosis de cemento de $425 \mathrm{kgf} / \mathrm{m}^{3}$. Se tienen 151 puntos, a los cuales se ha ajustado primeramente una recta con el objeto de conocer su inclinación. Resulta una recta de pendiente positiva cuya expresión es:

$$
A i=0,0488 \cdot A d+2,297
$$

en que $\mathrm{Ai}$ : cantidad de aire incorporado, \%

Ad: dosis de aditivo, gramos/saco de cemento.

La expresión indica que la cantidad de aire incorporado es directamente proporcional a la dosis de aditivo. Para un hormigón fabricado sin aditivo incorporador de aire resulta una cantidad de $23 \mathrm{l} / \mathrm{m}^{3} \cdot(2,3 \%)$ de aire, valor exagerado para un hormigón corriente con árido de tamaño máximo de 2". La recta indicaria además que la cantidad de aire es siempre directamente proporcional a la dosis de aditivo, lo que no es cierto, ya que sobre cierto límite no es posible incorporar más aire.

Según esto se podria considerar como más representativa una parábola de la forma:

$$
A i=u \cdot A d^{v}
$$

en que $u$ y $v$ son constantes.

Resolviendo se logró encontrar la siguiente expresión:

$$
A i=\frac{A d^{0.50252}}{1,4762}
$$

La representación gráfica de la curva se ha dibujado en la Figura 7 en la que se han representado también los puntos. Para una dosis de aditivo de $0 \mathrm{~g} / \mathrm{saco}$, el modelo no tiene sentido pero, por ejemplo, para una dosis de $1 \mathrm{~g} /$ saco se obtiene $6,71 / \mathrm{m}^{3}(0,67 \%)$, cantidad muy similar al contenido de aire de un hormigón corriente con áridos de tamaño máximo de 2".

\subsection{RELACION ENTRE CANTIDAD DE AIRE INCORPORADO Y RESISTENCIA A COMPRESION DEL HORMIGON}

En el hormigón con aire incorporado la resistencia a compresión varía además con la cantidad de aire. Para hormigones fabricados con el mismo cemento, iguales dosis por metro cúbico, e igual razón agua-cemento, y manteniendo los demás componentes, la resistencia a compresión del hormigón debe decrecer con el aumento de la cantidad de aire incorporado. 


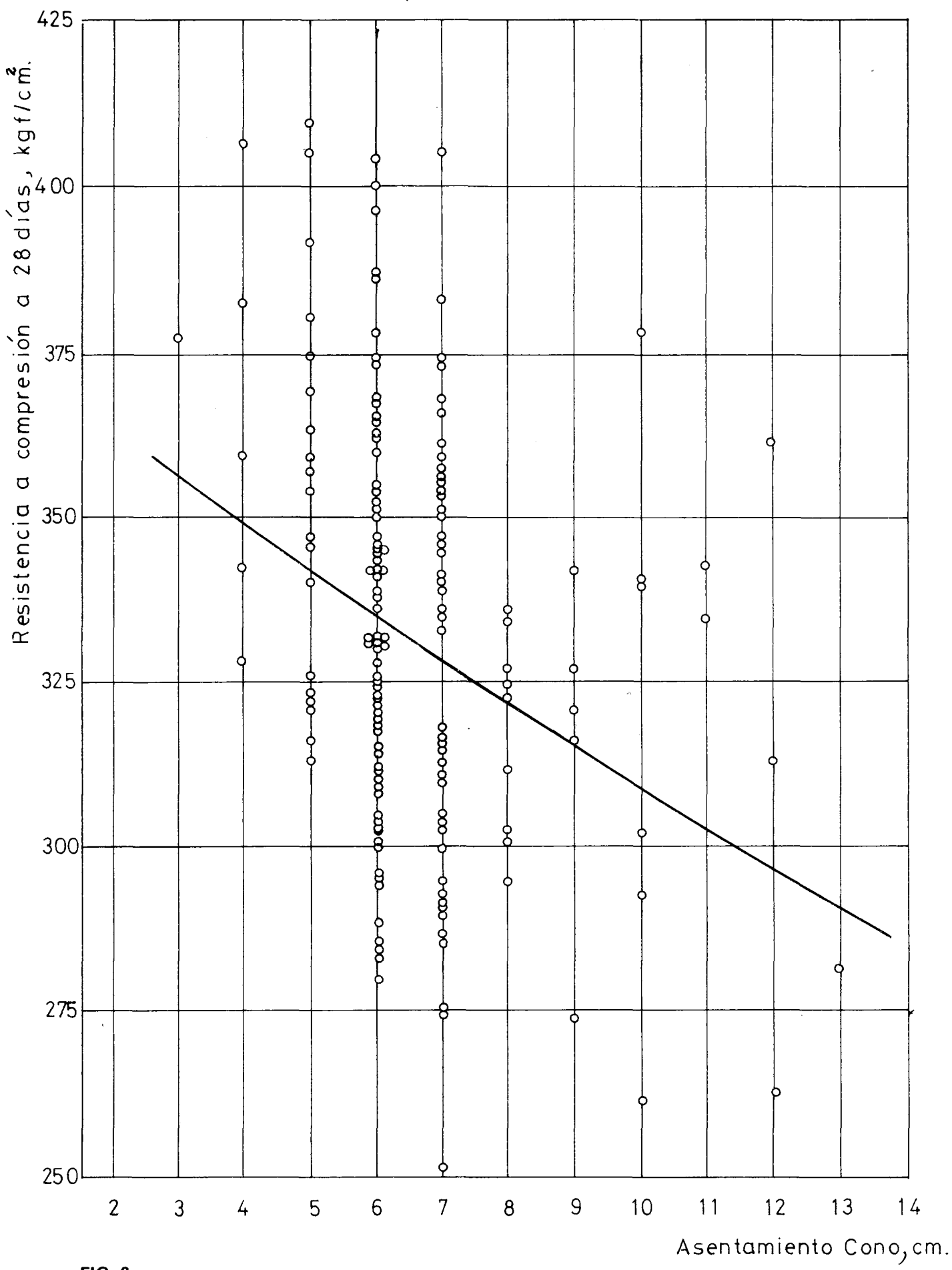

FIG. 6

En este caso se han considerado los resultados de resistencia a compresión de hormigones fabricados con dosis de cemento de $425 \mathrm{kgf} / \mathrm{m}^{3}$ y asentamientos en el cono de Abrams entre 6 y $8 \mathrm{~cm}$. Se tienen 120 valores los que se han representado en la Figura 8 . A los datos se ha ajustado en primer lugar una linea recta. La recta obtenida tiene la siguiente expresión:

$$
R_{28}=-29,448 \cdot A i+441,1 \quad \text { (curva 1) }
$$

en que $R_{28}$ : resistencia a compresión a 28 días, $\mathrm{kgf} / \mathrm{cm}^{2}$

Ai: cantidad de aire incorporado, \% 
La pendiente negativa de la recta verifica que existe disminución de resistencia a compresión del hormigón al aumentar la cantidad de aire incorporado.

Por otra parte hemos buscado una relación de la forma:

$$
\mathrm{R}_{28}=\frac{\mathrm{D}}{\mathrm{E}^{A i}}
$$

en que $D$ y $E$ son constantes.

Esta curva tiene la siguiente expresión:

$$
R_{28}=\frac{466,2}{1,096^{A i}}
$$

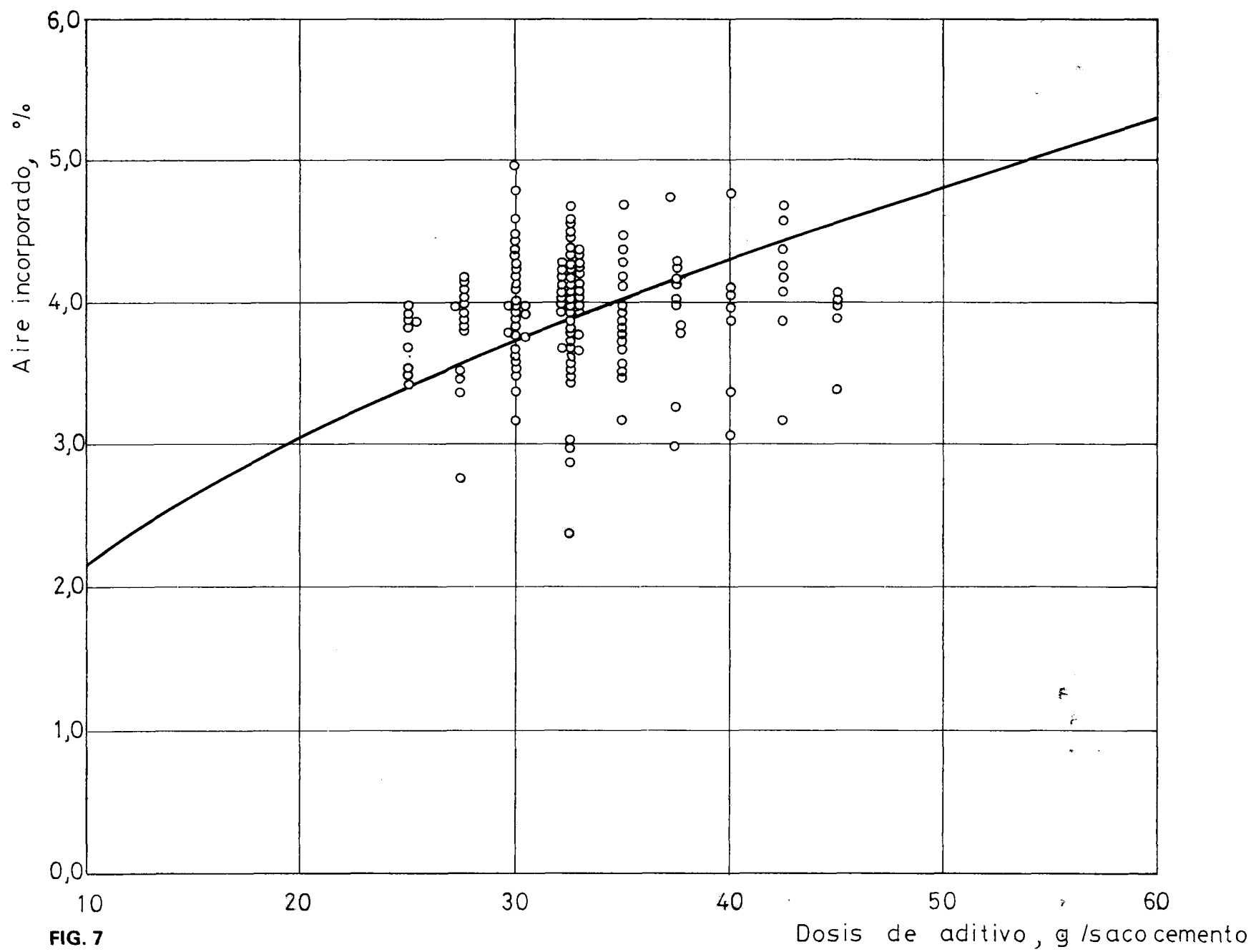




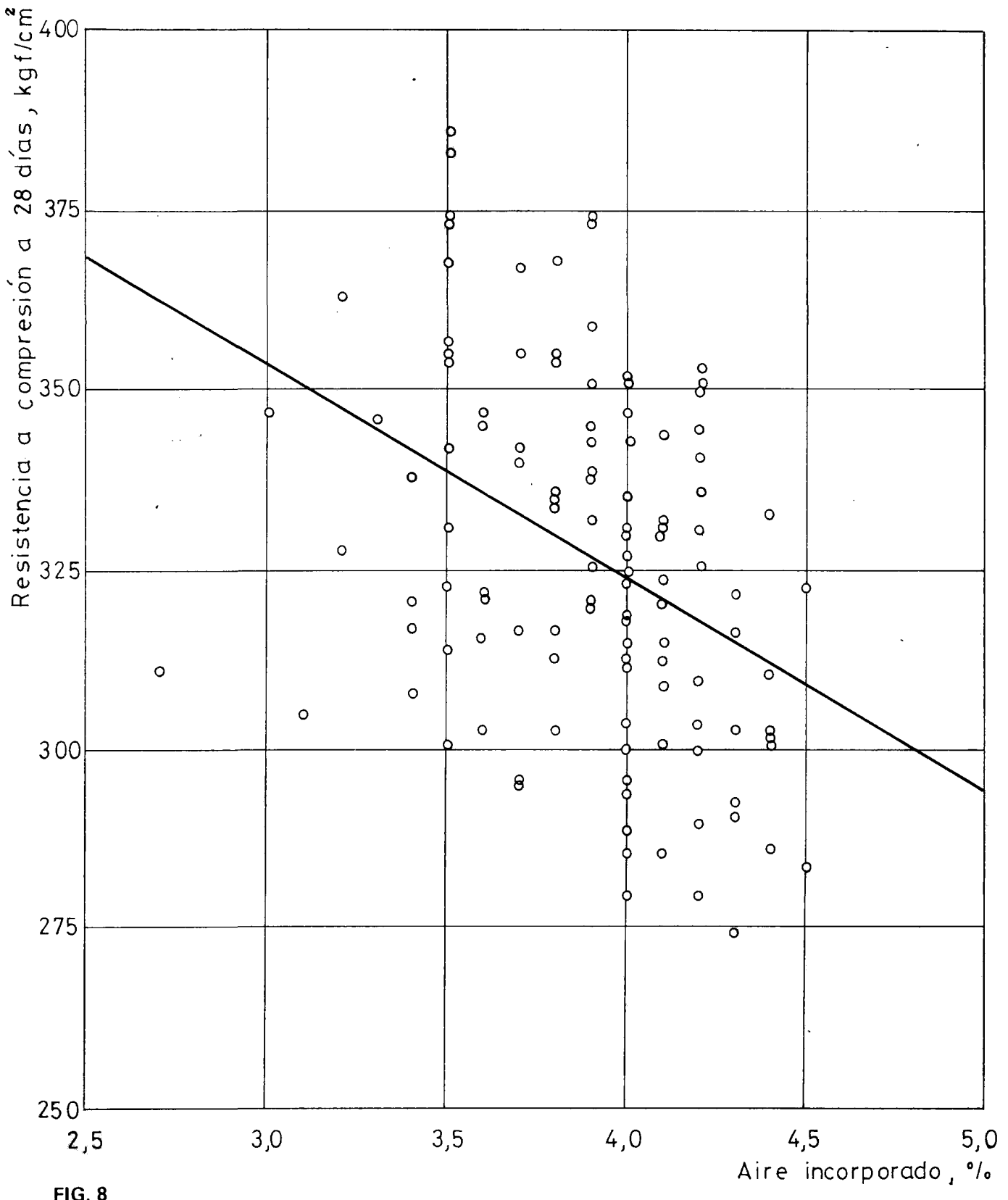

FIG. 8

pero dentro del intervalo que se encuentra la variable independiente (aire incorporado), ésta tiene la misma representación que la recta obtenida anteriormente. Por esta razón y por su mayor simplicidad se elige como relación resistencia vs aire incorporado la expresión de la curva 1.

Para un hormigón sin aditivo que contenga $0,67 \%$ de aire (hormigón corriente) la relación da una resistencia a compresión de $420 \mathrm{kgf} / \mathrm{cm}^{2}$, valor muy probable de obtener en un hormigón tradicional fabricado con cemento grado alta resistencia, en dosis de $425 \mathrm{kgf} / \mathrm{m}^{3}$ y asentamiento de cono de 6 a $8 \mathrm{~cm}$.

Finalmente de la misma expresión también se desprende que en los hormigones con aire incorporado se produce una disminución de la resistencia a compresión de aproximadamente $30 \mathrm{kgf} / \mathrm{cm}^{2}$ por cada $1 \%$ de aumento en el contenido de aire. 


\section{BIBLIOGRAFIA}

1. - AMMANN A., Le béton a áir occlus, Revue Sika N. ${ }^{\circ} 14$, junio, 1952

2. - ARCOS d'H C., Algunos aspectos de los hormigones con aire incorporado, Informes de la Construcción N. ${ }^{\circ}$ 286, Instituto Eduardo Torroja de la Construcción y del Cemento, Año XXIX, diciembre, 1976.

3.-CALLEJA J., Aditivos para el hormigón, Instituto del Cemento Portland Argentino, serie R, N. ${ }^{\circ} 57,1971$

4.-DE LA PEÑA C., La docilidad del hormigón y su medida, Instituto Técnico de la Construcción y del Cemento, Madrid, 1951

5. - HALD A., Statistical theory with engineering application, N. Y. John Wiley and Sons, 1: 52.

6. - HUMMEL A., Prontuario del hormigón, ETA, Barcelona, 1966.

7.-MARTIN B. and HAYNES R., Theoretical analysis of the effects of air voids in concrete, Journal of $\mathrm{ACl}$, vol. $68, \mathrm{~N} \cdot{ }^{\circ} 1$, enero, 1971.

8.-POPOVICS S., Effect of porosity on the strength of concrete, Journal of Materials, vol, 4, N. ${ }^{\circ} 2$, junio, 1969.

9. - SCRIPTURE E. and LITWINOWICS F., Some factors affecting air entraining, Journal of $\mathrm{ACl}$, febrero, 1949

10. - VARIOS AUTORES., Origin evolutions and effects of the air void system in concrete, part 1,2 y 3 , Journal of $A C l$, vol. 30, 1958.

11.-VENUAT M., Adjuvant et traitement des mortiers et betons, Centre D'Etudes et de Recherches de l'Industrie des Liants Hydrauliques, Paris, 1971.

12. - VENUAT M., traducción por PIÑEIRO M., Elementos de estadística, ejemplos de utilización para el cemento, IDIEM, Informe Técnico $N{ }^{\circ} 6,1963$

13. - WALKER S., Studies of concrete containing entraining-air, Journal of $\mathrm{ACl}, \mathrm{vol} .17, \mathrm{~N} .^{\circ} 6$, junio, 1946.

\section{résumé}

RESULTATS D'UN OUVRAGE CONSTRUIT EN BETON A AIR OCCLUS

Carlos Arcos d'Hainaut, chercheur IDIEM

Dans cet article, l'auteur présente une analyse des résultats d'un ouvrage entièrement construit en béton à air occlus. Les données analysées, qui correspondent aux résultats obtenus lors du contrôle de l'ouvrage, se trouvent dans les certificats officiels délivrés par IDIEM. On a obtenu quelques rapports généraux entre les facteurs et les coports generaux entre les facteurs et les clus qui reflètent les conditions de travail usuelles et qui peuvent être étendus aux bétons à air occlus en général.

\section{summary}

RESULTS IN A CONSTRUCTION JOB USING CONCRETE CONTAINING ENTRAINING-AIR

\section{Carlos Arcos d'Hainaut, Researcher} IDIEM

This paper presents the analysis of the results obtained in a construction iob where only concrete containing entraining-air was used. The analysed data are from result obtained during construction control procedures and are covered by official certificates issued by IDIEM. Some general relationship have been obtained between the factors and effects of the components of containing entraining-air reflecting usual working conditions and these can be applied to othe concretes containing entraining-air in general

\section{zusammenfassung ERGEBNISSE EINES BAUS AUS BETON MIT EINGESCHLOSSENER LUFT \\ Carlos Arcos d'Hainaut, Forscher IDIEM}

Diese Arbeit zeigt die Untersuchung der Ergebnisse eines Baus, der insgesamt aus Beton mit eingeschlossener Luft hergestellt wurde. Die untersuchten Angaben entsprechen den Ergebnissen, weiche während der Prüfungen der Bauarbeiten erhalten wurden und sind in den offiziellen, vom IDIEM veröffentlichen Bescheinigungen enthalten. Es wurden mehrere allgemeine Beziehungen zwischen den Faktoren und Wirkungen de Komponenten im Beton mit eingeschlossener Luft erhalten, welche die üblichen Arbeitsbedingungen wiedergeben und allgemein auf die Betonklassen mit eingeschlossener Luft ausgedehnt werden können. 